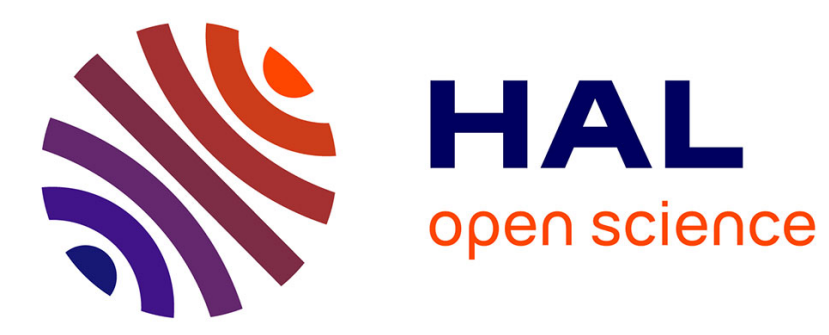

\title{
In pursuit of increasingly linear loudspeaker motors
}

Benoit Merit, Guy Lemarquand, Valérie Lemarquand

\section{To cite this version:}

Benoit Merit, Guy Lemarquand, Valérie Lemarquand. In pursuit of increasingly linear loudspeaker motors. IEEE Transactions on Magnetics, 2009, 45 (6), pp.2867. hal-00413418

\section{HAL Id: hal-00413418 https://hal.science/hal-00413418}

Submitted on 4 Sep 2009

HAL is a multi-disciplinary open access archive for the deposit and dissemination of scientific research documents, whether they are published or not. The documents may come from teaching and research institutions in France or abroad, or from public or private research centers.
L'archive ouverte pluridisciplinaire HAL, est destinée au dépôt et à la diffusion de documents scientifiques de niveau recherche, publiés ou non, émanant des établissements d'enseignement et de recherche français ou étrangers, des laboratoires publics ou privés. 


\title{
In pursuit of increasingly linear loudspeaker motors
}

\author{
B. Merit ${ }^{1,2}$, G. Lemarquand ${ }^{2}$, Member, IEEE, and V. Lemarquand ${ }^{2}$ \\ ${ }^{1}$ Orkidia Audio SARL, Av. Larramendy, 64500 St Jean de Luz, France \\ ${ }^{2}$ LAUM, CNRS, Université du Maine, Av. O. Messiaen, 72085 Le Mans, France
}

In this paper, analytical calculations are used to study existing loudspeaker motors totally made of permanent magnets. When the iron is removed from a loudspeaker, Eddy currents and reluctant effects are also removed, improving the linearity of the loudspeaker. Performances of each proposed magnetic motor structures are compared, from the intensity of their created induction to their ability to respond as an accurate image of the driving current. For a given structure, the authorized excursion length on which the loudspeaker motor is linear is a criterion for limiting its frequency range. The good understanding of the behavior of any magnets assemblies lead to a so called "linear structure", whose volume is optimized and able to create a very uniform induction, along a distance range that can be chosen. When this is achieved, the force that makes the moving part of the loudspeaker oscillate becomes proportional to the current running through its voice-coil. Thus, this loudspeaker motor is linear as well as using a reduced magnets volume, and can be applied to all frequency ranges.

Index Terms-Analytical calculations and optimizations of annular structures totally made of permanent magnets, linear loudspeaker motor.

\section{INTRODUCTION}

$\mathrm{T}$ HE DRAWBACKS due to the iron in loudspeaker motors have already been identified [1,2]. Iron is the source of Eddy currents, and creates the additional so called reluctant force, proportional to the square of the current! Moreover, when the magnetic field seen by the voice-coil depends on the axial position in the airgap, the motion force becomes also nonlinear. The iron in loudspeaker motors always leads to this dependence. The linearity of a loudspeaker needs the removal of the iron.

Thanks to a price decrease, motors totally made of permanent magnets become more and more interesting $[2,3,4]$. Searching for even more linear and accurate loudspeakers, the last problem to solve when iron is removed is to make uniform the radial magnetic induction seen by the coil along its entire trajectory [5].

This paper studies ironless structures, with the aim to compare them and to understand them, and paying attention to the linearity of the loudspeaker rather than its efficiency. To consider magnets that exist in the commerce, these annular structures only have rectangular cross sections. Then we propose a structure able to create well-controlled uniform magnetic field.

\section{ANALYTICAL STUDIES}

\section{A. Interest of analytical calculations}

Most work on magnetic structures is lead thanks to finite elements analysis. The magnetic field created by permanent magnets can be predicted whatever their shape or magnetization are. But it takes too long when searching for optimizing these structures. Thus, understanding and control of the behavior of such magnets is quite hard. This is very restrictive in the loudspeaker world, where the magnetic field

Digital Object Identifier inserted by IEEE has to be intense and uniform in a limited gap.

That is the reason why we have decided to calculate exactly the magnetic field created by any structures totally made of permanent magnets. Using a coulombian approach [6], the analytical resolution of (1) makes it possible to calculate the field $\vec{B}$ created at the point $\vec{r}$ by a magnet of magnetization $\vec{J}$ and volume $V_{m}$ bounded by the closed surface $S_{m}$ :

$$
\begin{aligned}
\vec{B}=-\frac{1}{4 \pi} \vec{\nabla}\left[\iint_{S_{m}}\left(\vec{J} \cdot d \vec{S}_{m}\left|\vec{r}-\vec{r}_{m}\right|\right)\right. & \\
& \left.+\iiint_{V_{m}}\left(-\nabla_{m} \vec{J} d V_{m}|| \vec{r}-\vec{r}_{m} \mid\right)\right] .
\end{aligned}
$$

In (1) the index $m$ refers to the coordinates of the considered magnet.

\section{B. Magnetic pole surface density}

This section only considers magnetic pole surface density, i.e. the first part of (1):

$$
\vec{B}_{S}=-\frac{1}{4 \pi} \vec{\nabla}\left[\iint_{S_{m}}\left(\vec{J} \cdot d \vec{S}_{m} /\left|\vec{r}-\vec{r}_{m}\right|\right)\right] .
$$

The three components of the magnetic field created by right angles magnets have already been analytically calculated [6].

But, especially because most loudspeakers are of revolution symmetry, it becomes important to estimate the effects due to the curvature of the magnets, whose magnetization can be axial or either radial. The induction created by annular structures, whatever the shape of their cross section is, can be semi-analytically calculated thanks to the approximations of [7] and [8]. But for annular magnets with rectangular cross sections, a complete analytical expression of this magnetic field can now be used [9].

\section{Magnetic pole volume density}

The calculations presented in reference [9] do not take into account the magnetic pole volume density. But we have shown [10] that this magnetic pole volume density can be omitted if the magnetic field is observed close to the faces of the ring. In loudspeakers, the voice-coil is rather close to the magnetic rings so that approximations of [9] are sufficient to predict the 
real behavior of magnetic structures. Nevertheless all results are verified thanks to semi-analytical resolutions $[6,10,11]$.

Thanks to the analytical resolution of (2), it is now possible to calculate quickly and without approximation the magnetic field created by these structures, with axial or radial magnetization. This tool is powerful and makes easier and faster the study of the influences of each annular magnets characteristic, especially their radius.

\section{BEHAVIOR OF IRONLESS STRUCTURES}

This section describes the three structures of ironless loudspeaker motor of Fig.1a, 1b and 1c. They are made of rings with rectangular section, whose magnetization $J$ or $j$ can be either radial or axial. For the studies, the magnetization $J$ is always equal to 1T. The point $O$ represents the location of the voice-coil in its rest position.

In the considered applications, the voice-coil oscillates in the direction $O z$ parallel to the revolution axis of the loudspeaker. The induction has to be uniform along this direction: the more uniform the induction, the more accurate the loudspeaker; and the more intense the magnetic induction, the more efficient the loudspeaker.

The induction is calculated on the $O z$ axis at a distance $a$ from the inner radius of the rings. This location corresponds to the mean value of the voice-coil thickness (the coil thickness is the radial length of the coil).

For the study, let us consider a parameter called $z_{\text {uni }}$. Given a structure, this parameter represents the axial distance across which the magnetic induction seen by the voice-coil has a peak-to-peak variation smaller than $1 \%$. Thus, across this distance, the induction is considered uniform. In classical loudspeakers, the induction variation easily exceeds $10 \%$ across a distance that corresponds to the trajectory of the voice-coil; that is why significant nonlinear effects are

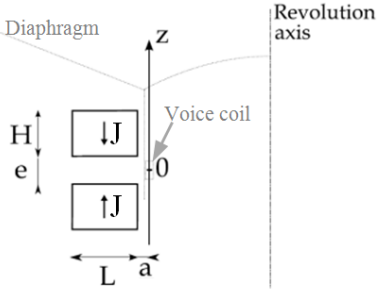

(a)

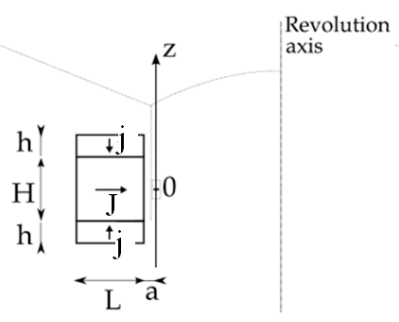

(c)

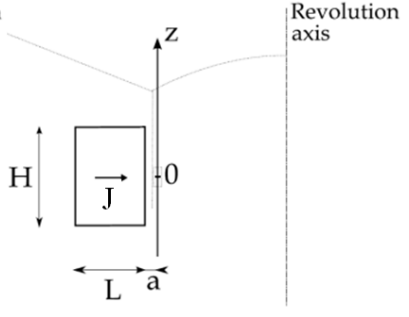

(b)

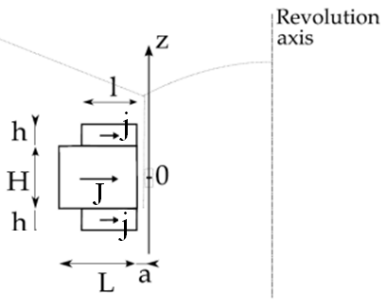

(d)
Fig. 1. The four studied ironless loudspeaker motor structures. a) two axially magnetized rings; b) one radially magnetized ring; c) one radially magnetized ring and two axially magnetized rings; d) three axially magnetized rings.

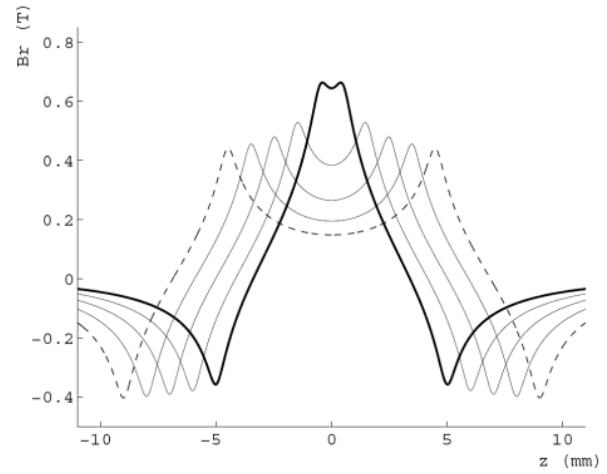

Fig. 2. Radial magnetic induction, $\mathrm{Br}(\mathrm{T})$, along the observation axis at a distance $a=0.3 \mathrm{~mm}$, created by the structure $(a)$ for $H=4.5 \mathrm{~mm}, L=9 \mathrm{~mm}$ and for various values of the distance between the magnets, from top to bottom, $e=1 \mathrm{~mm}$ (bold line), $3 \mathrm{~mm}, 5 \mathrm{~mm}, 7 \mathrm{~mm}$ and $9 \mathrm{~mm}$ (dashed line).

produced and it is important to search for structures that lead to more uniform induction.

\section{A. Two axially magnetized annular rings}

Fig.1a shows an ironless motor made of two axially magnetized concentric rings. These rings are positioned one above the other, at an axial distance $e$ from each other. They are separated by air and their respective magnetizations are in repulsion. Thus the magnetic flux in the coil is expected to be radial. This kind of structure has been patented by the Harman society [4].

Fig.2 shows how the created induction varies when the magnets deviate. This induction varies too much to obtain a significant $z_{u n i}$. When the magnets are close to each other, the induction can be intense, up to $0.6 \mathrm{~T}$, but then the possible linear coil displacement is very small. Thus it is quite clear that this structure should be dedicated to loudspeakers in which the voice-coil has very small displacements, so preferably to tweeters.

\section{$B$. One radially magnetized ring}

Let us consider the structure of Fig.1b. It is constituted by one radially magnetized ring facing the moving coil. Previous studies [2] on the induction created by a rectangular magnet show that a good combination between its dimensions can

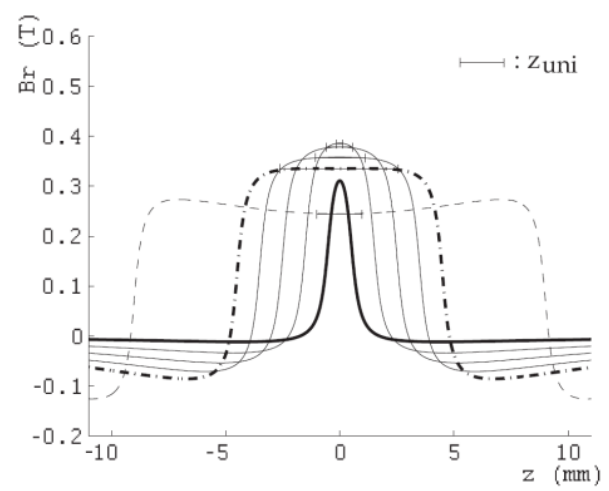

Fig. 3. Radial magnetic induction, $\mathrm{Br}(\mathrm{T})$, along the observation axis at a distance $a=0.3 \mathrm{~mm}$, created by the structure (b) for $L=9 \mathrm{~mm}$ and for various values of the height: $H=1 \mathrm{~mm}$ (bold line), then from top to bottom $H=3 \mathrm{~mm}$, 5mm, $7 \mathrm{~mm}, 9 \mathrm{~mm}$ (dot-dashed line) and $H=18 \mathrm{~mm}$ (dashed line). 

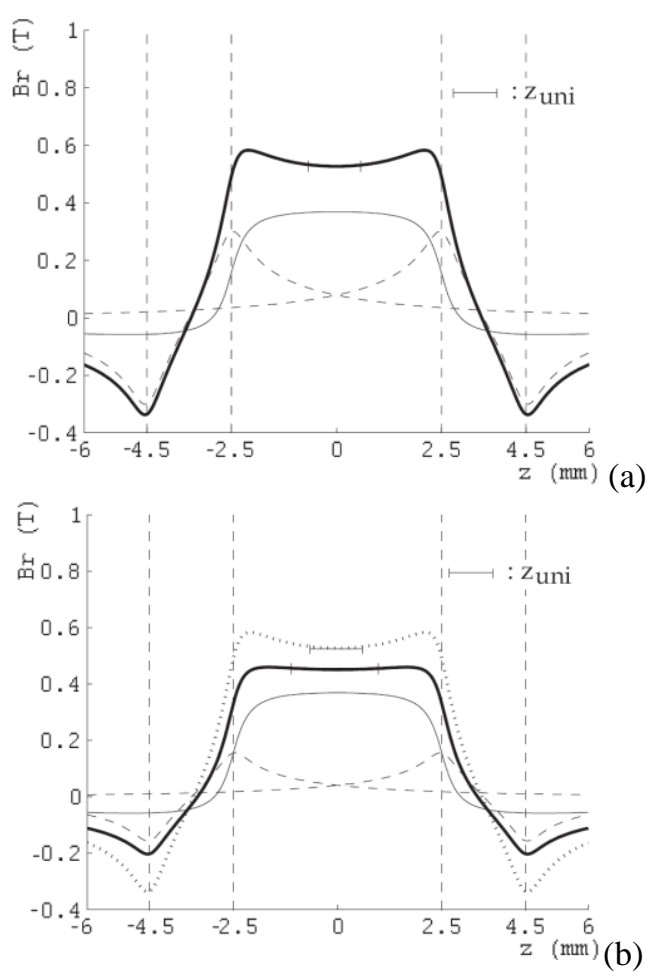

Fig. 4. (a) Radial magnetic induction, $\mathrm{Br}(\mathrm{T})$, along the observation axis at a distance $a=0.3 \mathrm{~mm}$, created by the structure (c) (bold line) with its central magnet with $H=5 \mathrm{~mm}, L=8 \mathrm{~mm}, J=1 \mathrm{~T}$ (full line) and its two external magnets with $h=2 \mathrm{~mm}, l=8 \mathrm{~mm}, j=1 \mathrm{~T}$ (dashed line). (b) Magnetization $j$ is lowered to $0.52 \mathrm{~T}$. The dotted-line remains the induction created by the patented structure where the magnetizations $J$ and $j$ are equal to 1T.

always lead to a quite uniform induction in front of the magnets. In the best case, i.e. when dimensions are analytically optimized, the axial distance $z_{u n i}$ is about $60 \%$ of the considered magnet height. The same conclusions are available for annular magnets with rectangular section.

Fig. 3 shows that, for a given length, the induction can be quite constant over an axial range if the magnet height is optimal (dot-dashed line in Fig.3). Thus this structure can be dedicated to every loudspeaker motors. But it generally needs significant volumes of magnets and its created induction intensity is modest.

Fig.3 also shows that small height magnets only act over short axial areas (see bold line in Fig.3). This localized action will be useful in further structures.

\section{Combination of the first two solutions}

The Sony society patented the structure of Fig.1c [3]. It is constituted by a stack of three rings of equal radial length. The central ring is radially magnetized while the external rings are axially magnetized. According to the patent, the magnetizations $J$ and $\mathrm{j}$ are equal. The magnetizations of the external rings help the total magnetic flux to close; so this structure combines the effects of the first two structures $(a)$ and $(b)$. That is why it creates high magnetic induction levels with improved uniformity compared to the structure $(a)$, while using relatively small volume of magnets.

Fig.4a shows the induction calculated along the observation axis created by the whole structure (bold line) and by the separated elements. The induction variation along the $O z$ axis over a range corresponding to the height of the central magnet represents $19 \%$ of the central value $(0.5 \mathrm{~T})$. Because of this significant variation, $z_{u n i}$ is only $1.28 \mathrm{~mm}$, that is $14 \%$ of the total height of the structure. Note that the induction variation is $10 \%$ across the distance of $4.2 \mathrm{~mm}$ that separates the two maxima of this induction. The structure (c), which is $11 \%$ more compact than the previous optimal structure (b), produces a more intense induction but with a larger induction variation. The induction intensity increases by $34 \%$ while the distance $z_{\text {uni }}$ decreases by $76 \%$ compared to the optimal structure $(b)$.

In addition, Fig.4a shows the contribution of each ring to the total induction and especially that the external rings provide a really high magnetic induction compared to the induction provided by the central ring (see dashed line in Fig.4a). Fig.4b shows the result when the external magnetizations are lowered to $0.52 \mathrm{~T}$. In this case $z_{\text {uni }}$ equals $2.1 \mathrm{~mm}$, and now the induction variation is only $1.8 \%$ across the $3.4 \mathrm{~mm}$ distance that separates the two maxima of this induction. The uniformity improvement is significant but leads to an $8 \%$ decrease of the total induction.

One has to note that this structure should not be used for low frequency drivers. When the intended $z_{\text {uni }}$ increases (over $10 \mathrm{~mm}$ ), the two external magnets do not contribute to increase the induction intensity anymore. In this frequency range, the structure $(c)$ would act like the structure $(b)$ while being more expensive.

\section{LINEAR STRUCTURE}

The structure of Fig.1d goes further in the idea of using magnets whose magnetizations differ in order to give the intended flat shape to the observed induction. It is constituted by a stack of three radially magnetized rings. It combines the higher magnetization of the external rings and their small height to use their much localized action (as described in Fig.3). Contrary to the previous structure (c), the external rings are only aimed to compensate the bell shape of the induction created by the central ring; that is why it creates less intense inductions.

Fig.5a shows the contribution of each ring and the resulting induction (bold line). The set of dimensions is chosen in order to have the same magnet section as the one of the previous optimal structure $(b)\left(81 \mathrm{~mm}^{2}\right)$. In this case, the performance improvement compared to the structures $(b)$ is not significant. But the structure $(d)$ can be optimized. Indeed, the distance $z_{u n i}$ can always correspond to the dimension $H$, without any influence on the induction intensity. The other dimensions and the magnetizations are calculated in order to optimize the volume of the whole structure, and the uniformity and the intensity of the magnetic field.

Thus, the geometry has been optimized in order to obtain an induction of equal intensity (0.33T) and having about the same $z_{\text {uni }}$ of $5.4 \mathrm{~mm}$. The optimal dimensions we find correspond to a more spacesaving structure whose total height 

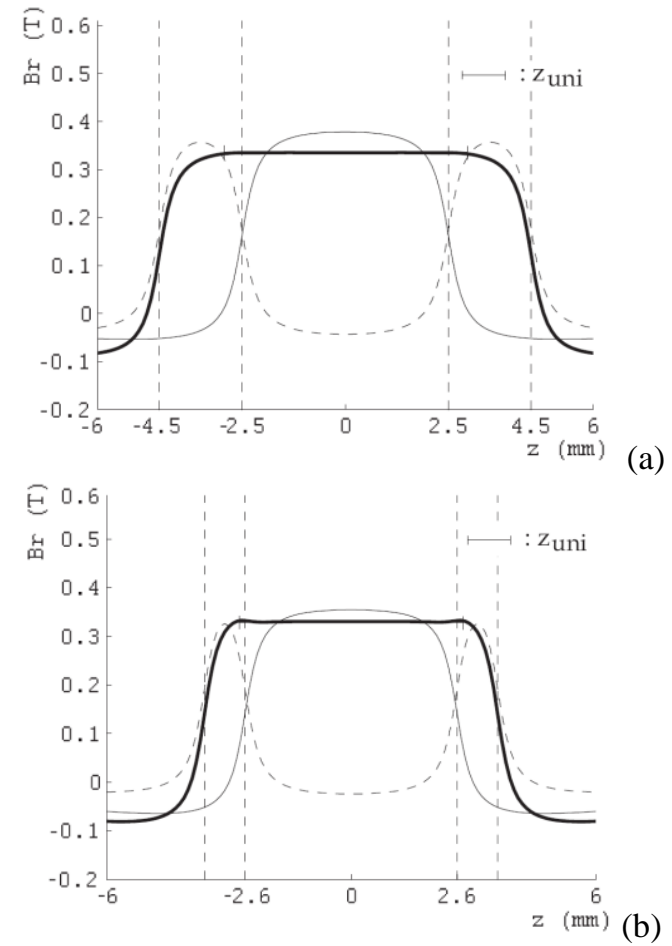

Fig. 5. Radial magnetic induction, $\mathrm{Br}(\mathrm{T})$, along the observation axis at a distance $a=0.3 \mathrm{~mm}$, created by the structure (d) (bold line) with : (a) its central magnet with $H=5 \mathrm{~mm}, L=9 \mathrm{~mm}, J=1 \mathrm{~T}$ (full line) and its two external magnets with $h=2 \mathrm{~mm}, l=9 \mathrm{~mm}, j=1.01 \mathrm{~T}$ (dashed line); (b) its central magnet with $H=5.2 \mathrm{~mm}, L=7 \mathrm{~mm}, J=1 \mathrm{~T}$ (full line) and its two external magnets with $h=1 \mathrm{~mm}, l=8.5 \mathrm{~mm}, j=1.09 \mathrm{~T}$ (dashed line).

is $7.2 \mathrm{~mm}$ (Fig.5b). The total section of this optimized structure $(d)$ is $53.4 \mathrm{~mm}^{2}$ ( $34 \%$ decrease compared to the previous structure). As a matter of fact, one can easily understand the benefit in linearity and in volume compared to the structure $(c)$, despite the loss of induction intensity. With about a 35\% decrease of the induction intensity, the volume of the structure $(d)$ is half the volume of the structure $(c)$ to obtain a uniform induction over the same $\mathrm{z}_{\mathrm{uni}}$ as the structure $(c)$.

As a result, the structure $(d)$ leads to very compact devices that have a very uniform induction over a range that can be chosen. This is also applicable when searching for higher distances $z_{\text {uni }}$, i.e. for low frequency loudspeakers. Fig.6 shows the induction created by both structures $(b)$ and $(d)$, whose volumes are optimized in order to obtain the same $z_{\text {uni }}$ equal to $40 \mathrm{~mm}$, with the same created induction intensity. The volume of $(d)$ is $20 \%$ lower than the one of the structure $(b)$.

Thus, since voice-coils can easily remain in a uniform induction, the structure $(d)$ leads to highly linear loudspeaker motors and represents a real benefit in space occupation.

\section{CONCLUSION}

Analytical calculations of the induction created by structures totally made of permanent magnets make it possible to only concentrate in an automatic optimization of one parameter as $\mathrm{z}_{\text {uni. }}$. This parameter represents the axial distance across which the induction is considered uniform; the uniformity of the induction being related to the linearity of the loudspeaker motor.

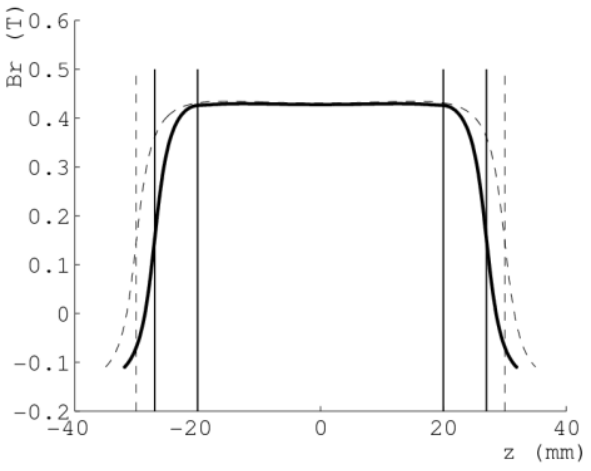

Fig. 6. Radial magnetic induction, $\mathrm{Br}(\mathrm{T})$, along the observation axis at a distance $a=2 \mathrm{~mm}$, created by the structure (d) (bold line) - with its central magnet with $H=40 \mathrm{~mm}, L=28 \mathrm{~mm}, J=1.43 \mathrm{~T}$ (full line) and its two external magnets with $h=7 \mathrm{~mm}, l=17 \mathrm{~mm}, j=1.49 \mathrm{~T}$ (dashed line) - and by the structure (b) (dashed line) with $H=60 \mathrm{~mm}$ and $L=46 \mathrm{~mm}$ and $J=1.43 \mathrm{~T}$. The linear excursion of the voice-coil is $\pm 20 \mathrm{~mm}$.

So the parameter $z_{\text {uni }}$ should be directly related to the frequency range of a loudspeaker. Thus, the structure (a) should only be dedicated to tweeters, while structure $(c)$ can also be dedicated to high-medium frequency loudspeakers. Structures $(b)$ and $(d)$ are more universal, but create less intense inductions. For very small displacements, the dimensions of the external rings of the structure $(d)$ become negligible to compensate the bell-shaped induction created by the central ring. So the structure $(b)$ is sufficient compared to the structure $(d)$. But as soon as the intended $z_{\mathrm{uni}}$ increases, the structure $(d)$ represents a real benefit in linearity and in space occupation compared to the other structures.

\section{REFERENCES}

[1] J. Vanderkooy, "A model of loudspeaker driver impedance incorporating Eddy currents in the pole structure", J. Audio Eng. Soc., 37, March (1989).

[2] G. Lemarquand, "Ironless loudspeakers", IEEE Trans. Magn.. vol. 43, pp.3371-3374, August 2007.

[3] Y. Ohashi, "Magnetic circuit and speaker", Patent EP 1553802 A2, 2005.

[4] W. House et al., "Transducer motor assembly", US Patent 5142 260, 1992

[5] W. Klippel, Tutorial "Loudspeaker Nonlinearities- Causes, Parameters, Sysmptoms", J. Audio Eng. Soc. 54, No 10, pp. 901-939, October 2006.

[6] H. Rakotoarison, J-P Yonnet, B. Delinchant, "Using coulombian approach for modeling scalar potential and magnetic field of a permanent magnet with radial polarization", IEEE Trans. Magn., vol. 43, pp. 1261-1264, April 2007.

[7] F. Bancel, G. Lemarquand, "Three-dimensional Analytical Optimization of Permanent Magnets Alternated Structure”, IEEE Trans. Magn., vol. 34, pp. 242-247, January 2008.

[8] J.-P.Yonnet, "Rare-Earth Iron Permanent Magnets", Oxford, U.K.: Oxford Science, 1996, ch.8 Magnetomechanical devices.

[9] R. Ravaud et al., "Analytical calculation of magnetic field created by permanent magnet rings", IEEE Trans. Magn., vol. 44, pp. 1982-1989, July 2008.

[10] R. Ravaud et al., "Influence of the magnetic pole volume density on the magnetic field created by ring permanent magnets whose polarization is radial", IEEE Trans. Magn., submitted for publication on June 2008.

[11] E. P. Furlani, S. Reznik, and A. Kroll, "A three-dimensional field solution for radially polarized cylinders," IEEE Trans. Magn., vol. 31, pp. 844-851, January 1995.

Manuscript received October 16, 2008. Corresponding author: B. Merit (email: benoit.merit.etu@univ-lemans.fr. 